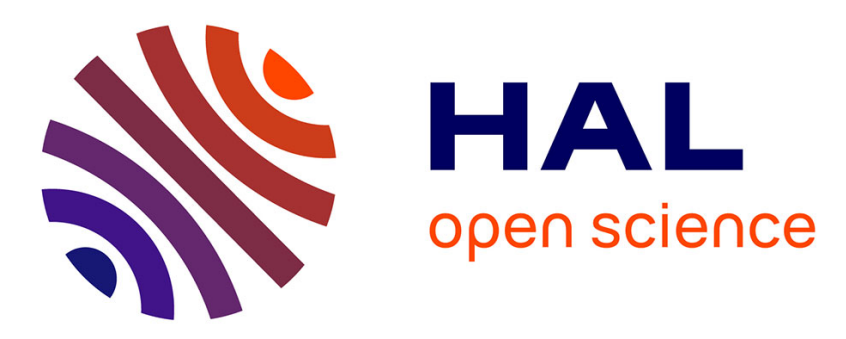

\title{
Use of Distributed Fiber Optic Sensors to Detect Damage in a Pavement
}

Xavier Chapeleau, Juliette Blanc, Pierre Hornych, Jean-Luc Gautier, Jean Carroget

\section{- To cite this version:}

Xavier Chapeleau, Juliette Blanc, Pierre Hornych, Jean-Luc Gautier, Jean Carroget. Use of Distributed Fiber Optic Sensors to Detect Damage in a Pavement. EWSHM - 7th European Workshop on Structural Health Monitoring, IFFSTTAR, Inria, Université de Nantes, Jul 2014, Nantes, France. hal-01022035

\section{HAL Id: hal-01022035 \\ https://inria.hal.science/hal-01022035}

Submitted on 10 Jul 2014

HAL is a multi-disciplinary open access archive for the deposit and dissemination of scientific research documents, whether they are published or not. The documents may come from teaching and research institutions in France or abroad, or from public or private research centers.
L'archive ouverte pluridisciplinaire HAL, est destinée au dépôt et à la diffusion de documents scientifiques de niveau recherche, publiés ou non, émanant des établissements d'enseignement et de recherche français ou étrangers, des laboratoires publics ou privés. 


\title{
USE OF DISTRIBUTED FIBER OPTIC SENSORS TO DETECT DAMAGE IN A PAVEMENT
}

\author{
Xavier Chapeleau ${ }^{1}$, Juliette Blanc ${ }^{2}$, Pierre Hornych ${ }^{2}$, Jean-Luc Gautier ${ }^{3}$, Jean Carroget ${ }^{3}$ \\ ${ }^{I}$ IFSTTAR, COSYS/II, Route de Bouaye - CS4, 44344 Bouguenais, France \\ ${ }^{2}$ IFSTTAR, MAST/LAMES, Route de Bouaye - CS4, 44344 Bouguenais, France \\ ${ }^{3}$ COLAS, Campus scientifique et technique - 4 rue Jean Mermoz, 78772 Magny-les-Hameaux, \\ France
}

xavier.chapeleau@ifsttar.fr, juliette.blanc@ifsttar.fr,pierre.hornych@ifsttar.fr

\begin{abstract}
This paper presents the feasibility of damage detection in the asphalt pavements by embedded fiber optics as a new non-destructive inspection technique. The distributed fiber optic sensing technology called "Rayleigh technique" was used in this study. The main advantage of this technique is that it allows to measure strains over long length of fiber optic with a high spatial resolution, less than $1 \mathrm{~cm}$. By comparing strain profiles measured at different time, an attempt was made to link strain changes with the appearance of damage (cracking) in the pavement. This non-destructive method was evaluated on the IFSTTAR accelerated pavement testing facility, in a bituminous pavement. In our experimentation, the optical fibers were placed near the bottom of the asphalt layer. The application of 728000 heavy vehicle loads $(65 \mathrm{KN}$ dual wheel loads) was simulated in the experiment. Optical fiber measurements were made at regular intervals and surface cracking of the pavement was surveyed. After some traffic, a significant increase of strains was detected by the optical fibers at different points in the pavement structure, before any damage was visible. Later, cracking developed in the zones where the strain profiles were modified, thus indicating a clear relationship between the increased strains and crack initiation. These first tests demonstrate that distributed fiber optic sensor based on Rayleigh sensing technique can be used to detect crack initiation and propagation in pavements, by monitoring strain profiles in the bituminous layers.
\end{abstract}

KEYWORDS: Distributed fiber optic sensor, asphalt concrete pavement, cracks, nondestructive inspection.

\section{INTRODUCTION}

The fiber optic sensor technologies offer interesting benefits when compared with traditional sensors. The main advantages are small size, electrically passive operation, electromagnetic immunity, flexibility, corrosion resistance, etc. Moreover, the technologies of fiber optic sensors become more and more efficient and reliable. Fiber optic sensors can be used to perform local or distributed measurements with precision in a wide range of strain and temperature. Several of them have already been used for structural health monitoring and surveillance with positive results. The most promising technology is the distributed sensing technique, which can be used to make practically continuous measurements over long lengths of fiber.

\section{FIBER OPTIC SENSOR SELECTED FOR THE STUDY}

The "Rayleigh sensing technique" is one of the distributed fiber optic sensing techniques having the highest spatial resolution (less than $1 \mathrm{~cm}$ ). It is sensitive to both strain and temperature. This 
distributed sensing technique is based on the measurement of the backscattering of light by random fluctuations of the refractive index profile along the length of the optical fiber. A strain (or temperature) profile can be obtained from two successive measurements of the Rayleigh signal, one acquired at an initial state and the second one measured at time $t$. The typical accuracy of the Rayleigh sensing technique is better than $\pm 1^{\circ} \mathrm{C}$ in temperature and $\pm 1 \mu \mathrm{m}$ in strain with a centimeter spatial resolution.

The idea, in this study, is to use the strain profile to detect cracks in pavements. If a crack occurs transversely to the fiber optic sensor, the strain transfer from the host material to the fiber optic will be modified locally. By comparing strain profiles measured at different times to detect strain changes along the fiber optic, internal damage (cracks) in the pavement should be detectable. This paper presents a first evaluation of this non-destructive damage detection method on the IFSTTAR accelerated pavement testing facility.

\section{DESCRIPTION OF THE EXPERIMENTATION}

\subsection{Accelerated pavement testing facility}

The pavement fatigue carrousel of IFSTTAR (Figure 1) is a large scale circular outdoor facility, unique in Europe by its size (120 m length, $6 \mathrm{~m}$ width) and loading capabilities [1]:

- maximum loading speed $100 \mathrm{~km} / \mathrm{h}$,

- loading rate 1 million cycles per month,

- 4 arms which can be equipped with different wheel configurations,

- lateral wandering of the loads to reproduce real traffic.

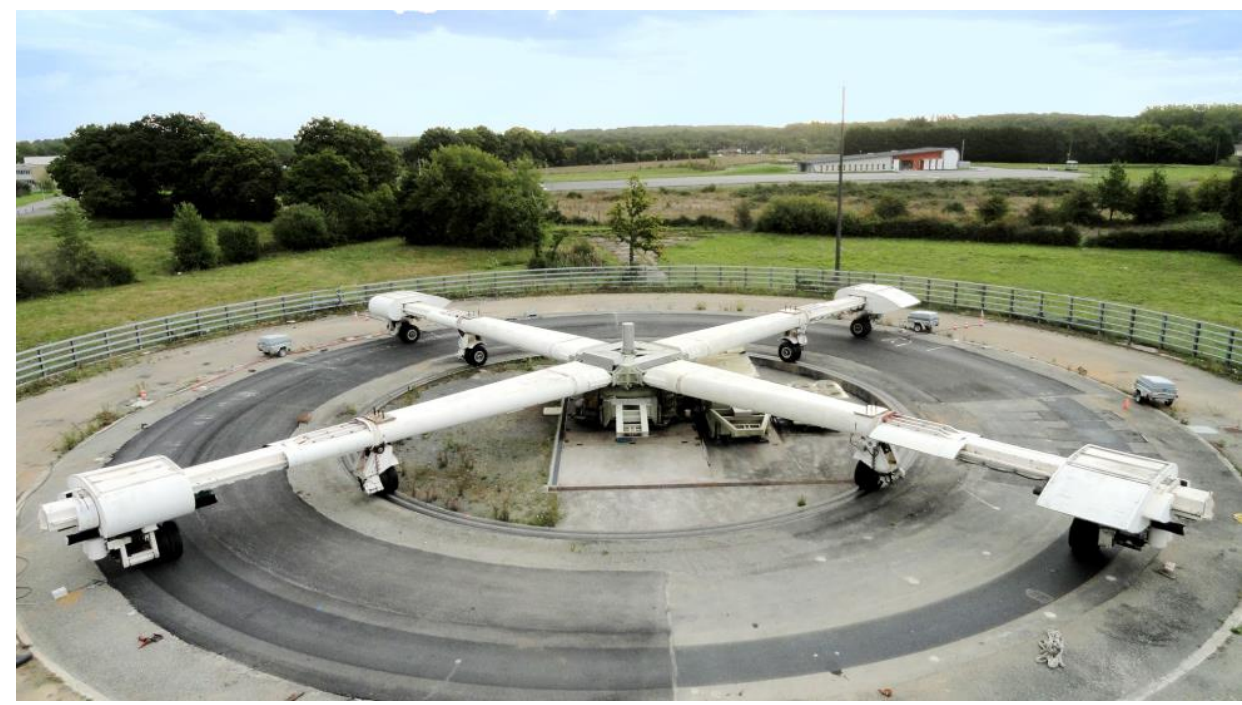

Figure 1: Accelerated pavement testing facility of IFSTTAR

\subsection{Fiber optic sensors and measurement system}

The Rayleigh sensing technique can be applied to standard fiber optic cables used for telecommunication systems. However, for engineering structures monitoring like pavements, special fiber optic cables designed for sensing measurement should be used. They must have high enough mechanical resistance for easy handling and to withstand the stresses experienced during the pavement construction process. For this experimentation, an optical fiber cable containing 6 fibers (only one fiber optic was used), protected by an epoxy overcoat and a hytrel jacket was selected. 
This cable is robust enough to be embedded in asphalt layers, while keeping a small diameter (2mm).

The measurements were made using a commercial device called "Optical Backscatter Reflectometer" (Luna's OBR). Strain or temperature profiles can be obtained from two measurements of Rayleigh signal. The measurement range is approximately $70 \mathrm{~m}$ with a measurement time less than $10 \mathrm{~s}$. This sensing technique has already been tested successfully for damage detection in composites laminates [2], for crack detection in concrete [3,4] and for structural tests of wind turbine blade [5]. Its main limitation is the measurement time, which allows making measurements only under static loads.

\subsection{Experimental pavement structure}

The fiber optic measurement technique was evaluated on a typical French low traffic pavement [6] consisting of $80 \mathrm{~mm}$ of high modulus asphalt, over a granular sub-base (300 mm thick, $300 \mathrm{MPa}$ of modulus), and a sandy subgrade soil.

To accelerate damage, artificial cracks were initiated in the pavement by inserting steel bars with different shapes (triangle, $\mathrm{T}$ shaped and flat) in the layer of asphalt. These bars were placed at the bottom of the asphalt layer, in the transverse direction, to initiate bottom-up cracking (Figure 2). The objective was to initiate cracks at an early stage, which would propagate transversely to the wheel path axis between the two bars of the same shape, placed $40 \mathrm{~cm}$ apart.

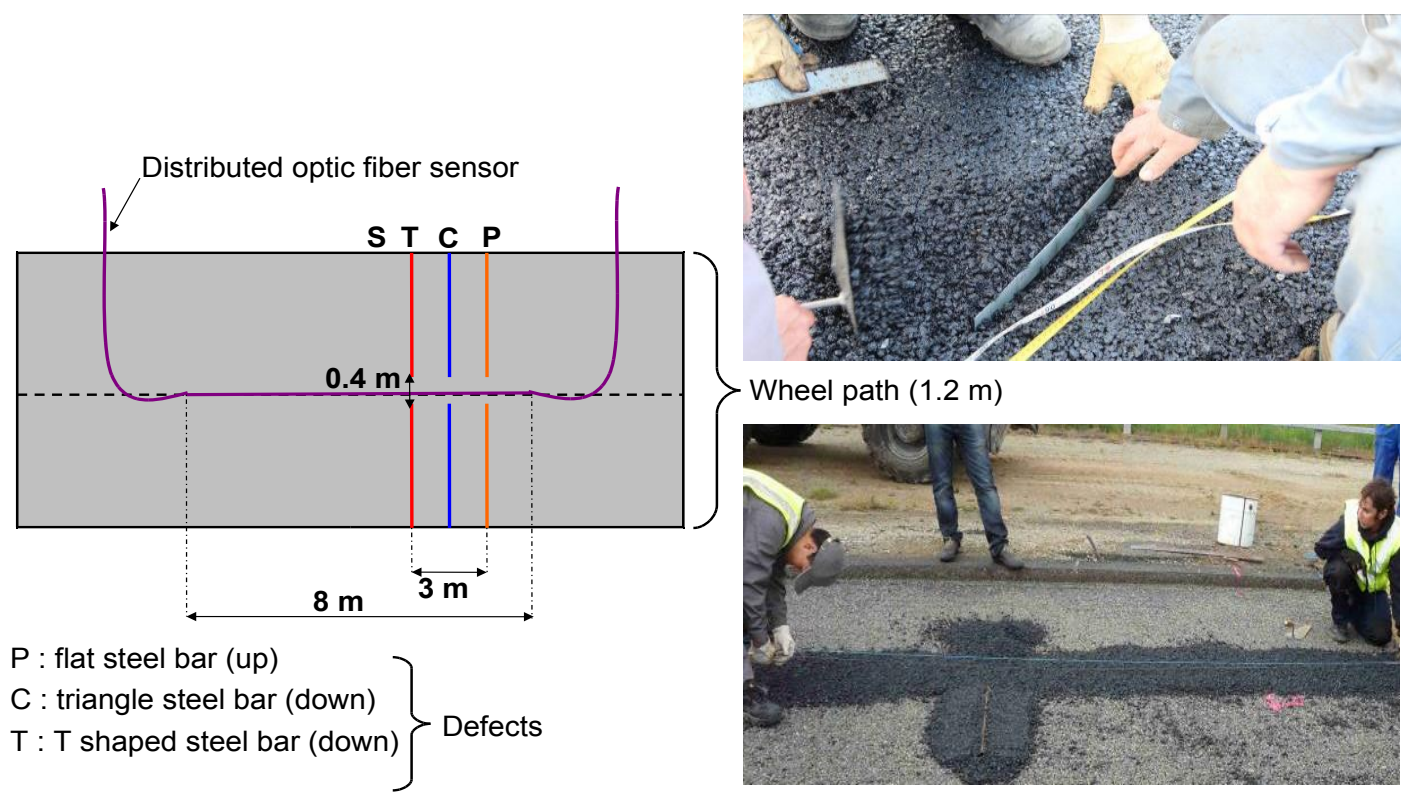

Figure 2: Plan of implementation of the steel bars and of the fiber optic cable

The distributed fiber optic sensor was placed in the wheel path axis of the pavement, over a length of $8 \mathrm{~m}$, in the zone containing the steel bars. The optical cable was put in place in three steps. First, a thin layer of asphalt (about $10 \mathrm{~mm}$ thick) was placed manually on the UGM foundation and compacted. The optical cable was put on this layer of asphalt (photo in the right corner of Figure 2) and covered manually by a thin layer of asphalt to maintain it in place. Finally, a paver and compactor were used to place an asphalt layer of $80 \mathrm{~mm}$. By this process, the optical cable was integrated in the pavement at $70 \mathrm{~mm}$ depth, near the bottom of the asphalt layer. 


\section{EXPERIMENTAL PROTOCOL}

\subsection{Applied loads}

In this experimentation, the four arms of the pavement fatigue carrousel have been equipped with standard dual wheels, loaded at $65 \mathrm{kN}$ (standard French equivalent axle load). The tires used are Dunlop $1200 \mathrm{R} 20 \mathrm{SP} 321$, inflated at $850 \mathrm{kPa}$ and the loading speed was 6 rounds per minute (43 $\mathrm{km} / \mathrm{h}$ ). At the beginning of the experimentation (until 308000 load cycles) the dual wheels were loaded at $65 \mathrm{kN}$. Afterward, the loads on each arm were increased to $75 \mathrm{kN}$, to accelerate pavement damage.

For testing schedule reasons and maintenance operations of the pavement fatigue carrousel, the experimentation was conducted in several phases:

- between August 2012 and September 2012, until 308000 load cycles,

- between December 2012 and January 2013, until 525000 load cycles,

- between September 2013 and December 2013, until 728000 load cycles.

\subsection{Fiber optic measurements}

The fiber optic measurements were performed every 50000 load cycles and surface cracking of the pavement was surveyed at the same time. All the measurements were obtained using the same protocol:

1. A "reference" measurement of the Rayleigh signal was performed with no load applied on the area of the fiber optic embedded in the pavement.

2. Then, one of the four dual wheels $(65 \mathrm{kN}$ or $75 \mathrm{kN})$ of the pavement fatigue carrousel was placed at different positions above the fiber optic sensor (positions "P", "C", "T" and "S" on Figure 2), and a second measurement was made.

To minimize the influence of the viscoelastic asphalt material behavior (leading to creep of the material under static load), the same time interval was always kept between the application of the load and the Rayleigh signal measurement (one minute). In addition, the measurements at the different positions were performed, for each sequence, in less than 20 minutes, to avoid temperature variations.

A fiber optic measurement consists in recording the Rayleigh backscattered signal along the optical fiber. The distributed strains can be obtained from two measurements where one is used as reference. So there are different ways (based on the choice on the reference scan) to post-treat the Rayleigh signal measurements which were performed according to the protocol described previously:

- Method 1: This method consisted in comparing the measurements made at the same number of load cycles, under the application of a static wheel load, and with no load. The strain profiles obtained with this method represent the relative strain change between the "loaded" and "unloaded" state.

- Method 2: In this method, no load was applied, and the measurements made after a certain number of load cycles were compared with the "reference" measurement performed at the beginning of the experimentation (at zero load cycles). With this second method, the strain profiles obtained represent the evolution of strains along the optical fiber throughout the duration of the experiment.

\section{RESULTS AND DISCUSSION}

First of all, it is worth to underline that all the fiber optic cables were embedded successfully in the asphalt layer without any damage. The conditions of construction of the pavement fatigue carrousel were close to road or highway construction. The fiber optic cable has withstood the high temperature of the asphalt mix (about $170{ }^{\circ} \mathrm{C}$ ) and the compaction of the pavement layer. 
Nevertheless, we recognize that the level of compaction was slightly lower that in real pavement construction to avoid to damage irremediably the fiber optic cable. Note that in this experiment, we chose to test a low-cost fiber optic cable which was not specially designed to support compression. It would have been possible to find more resistant optic cable, but at a price per meter 3-5 times higher.
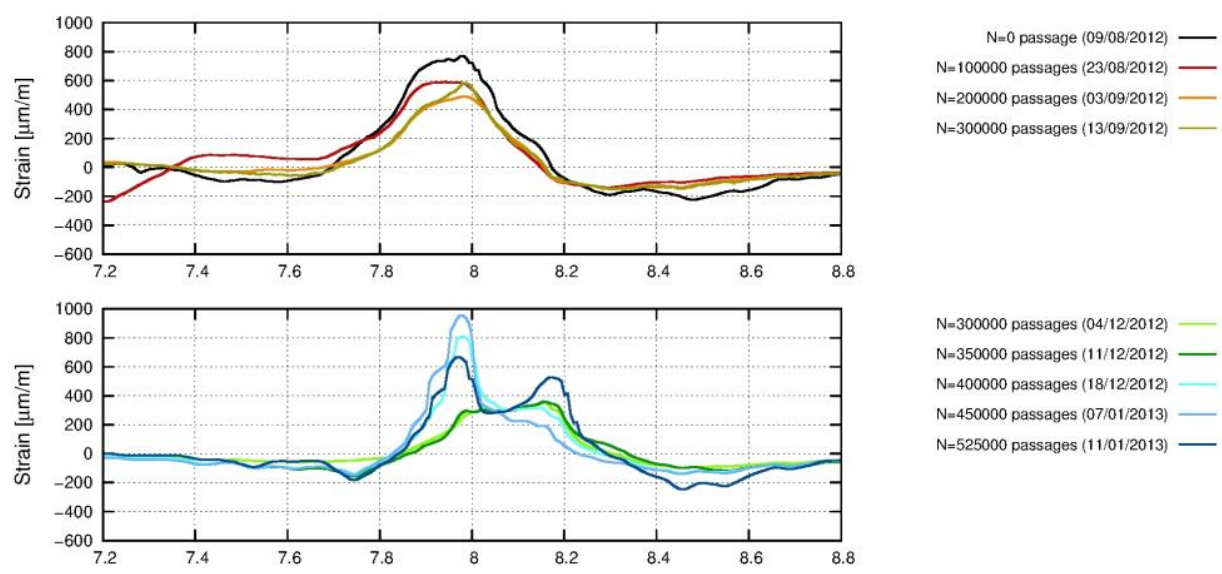

$N=300000$ passages $(04 / 12 / 2012)$ $\mathrm{N}=350000$ passages (11/12/2012) $\mathrm{N}=400000$ passages $(18 / 12 / 2012)$ $\mathrm{N}=450000$ passages $(07 / 01 / 2013)$ $\mathrm{N}=525000$ passages $(11 / 01 / 2013)$

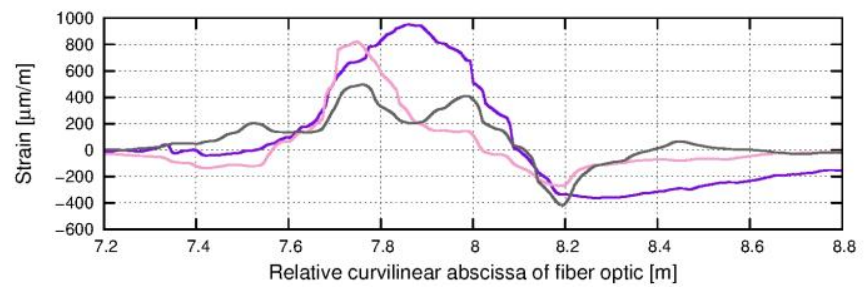

$\mathrm{N}=525000$ passages $(24 / 09 / 2013)$ $\mathrm{N}=625000$ passages $(30 / 10 / 2013)$ $\mathrm{N}=728000$ passages $(10 / 12 / 2013)$

Figure 3: Strains measured at position "P" at different numbers of load cycles

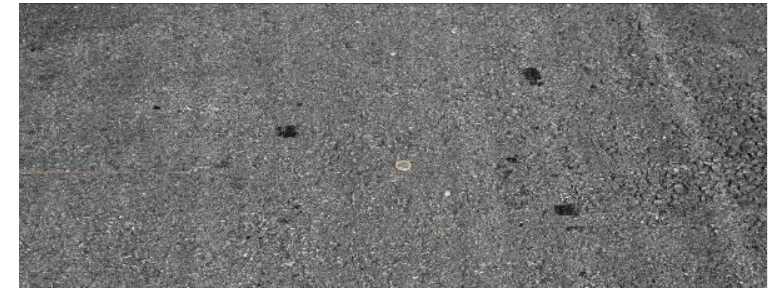

(a) At 300000 load cycles

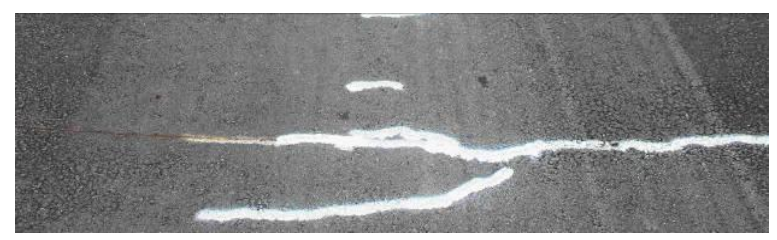

(c) At 525000 load cycles

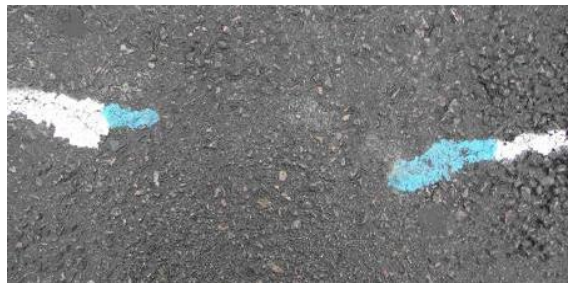

(b) At 450000 load cycles

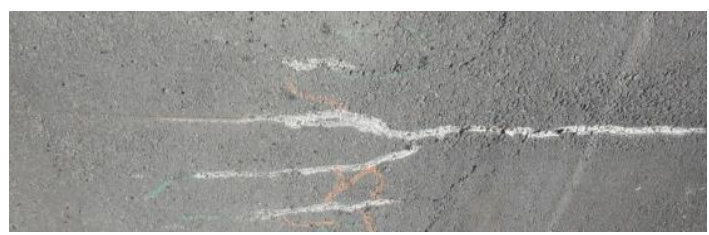

(d) At 728000 load cycles

Figure 4: Evolution of cracking of the pavement structure at position "P"

The first results presented in Figure 3 were obtained according to method 1 described previously. This figure shows the strain profiles measured at different numbers of load cycles, with a static load applied at the position "P", where the flat steel bars were placed to initiate cracking. As 
can be seen, all the strain profiles from the beginning of the experimentation to 300000 load cycles have the same shape. No significant change of width and maximum of the peak can be observed. The only difference is that the abscissa of the peaks is slightly shifted. It is due to the fact that the tire (the load) was not placed exactly at the same position for the different measurements. In addition to fiber optic measurements, regular visual inspections (Figure 4-a) did not permit to detect any cracks on the experimental pavement. So it seems logical to detect no change in the strain profile measurements.

The first cracks were detected on the surface of the pavement after 350000 load cycles. They appeared, as expected, at the ends of the steel bars placed in the pavement to initiate early cracks (Figure 4-b). At the same time, between 300000 and 350000 load cycles, the strain profile curves measured by the optical fibers started to change, the peak becoming wider and more flat in comparison with the previous measurements.

After 400000 and 450000 loads cycles, the maximum of the peak of the strain profile curves continued to increase and simultaneously, the surface crack propagated over the whole width of the pavement section. However, at the beginning, the crack remained very thin and difficult to detect by human eye. Then, at 525000 load cycles, the crack observed on the surface split in two cracks, (Figure 4-c) and at the same time, a second peak appeared on the strain profile. This result shows unequivocally the ability of the optic fiber to detect the initiation of cracks in the pavement. It is probable that the two peaks on the strain profile can be distinguished because the distance separating the two cracks is sufficient (about $20 \mathrm{~cm}$ ). The stress transfer from the crack to the sensor seems to occur over a length of approximately 20 centimeters.

After 500000 load cycles, the cracks still continued to grow, and news cracks appeared in the same area. At the end of the experimentation (728 000 load cycles), the pavement was severely damaged. As shown in figure Figure 4-d, at the end, the cracks were interconnected, creating a pattern called " alligator cracking". It is worth to note that the fiber optic cable continued to respond well despite the severe cracking of the pavement structure. However, the multiple cracks cannot be clearly distinguished, because the distance between them is less than the length of the stress transfer mechanism.

Similar results were obtained at the positions "C" and "T", where steel bars were also inserted in the pavement. The evolution of strain profiles at these positions was very similar to that obtained at position "P". These measurements were again consistent with observations of cracks at the surface of the pavement. All these results clearly demonstrate that distributed fiber optic sensors based on Rayleigh sensing technique can detect crack initiation and propagation by monitoring the shape of the strain profiles measured near the bottom of the pavement layer, at different times. However, the method of post-treatment of the Rayleigh signal measurements which was used to obtain the strain profiles presented above is not suitable for monitoring of long road sections. Indeed, this method requires applying a static load on the pavement which is very time consuming. For this reason, we have also tested another measurement method, which requires no load. With this method, The reference measurement (no load applied on the area of the fiber optic embedded in the pavement) used in the post-treatment of the fiber optic measurements was the reference measurement performed at the beginning of the experimentation (zero load cycles). The other measurements performed at different load cycles were then compared to this reference. With this procedure, it was possible to survey the evolution of strains along the optical fiber from the beginning to the end of the experimentation.

Figure 5 shows the strain profile curves obtained with the method described above. The first thing to underline is the very good repeatability of the strain profiles between 100000 and 300000 load cycles. The connections and disconnections of the fiber optic sensor to the interrogator imposed by the measurements at different days did not deteriorate the signal/noise ratio of the strain measurements. Moreover, the good repeatability of the strain profiles can be related to the fact that no cracks appeared until 300000 load cycles. At 350000 cycles (green curve), first significant changes can be observed on the strain profile. The positions where these changes occur $(x=8 \mathrm{~m}$ and 
$\mathrm{x}=9,5 \mathrm{~m}$ ) correspond well to the positions of the first cracks on the pavement, which were detected several thousand cycles later. At 400000 cycles, two strain peaks appear at position " $\mathrm{P}$ " $(\mathrm{x}=8 \mathrm{~m})$ corresponding to the two cracks observed close to this point.

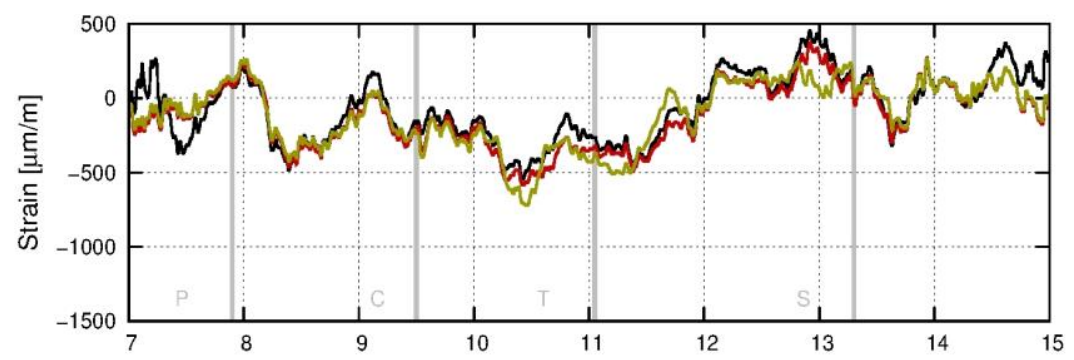

$\mathrm{N}=100000$ passages $(23 / 08 / 2012)$ $\mathrm{N}=200000$ passages $(03 / 09 / 2012)$ $\mathrm{N}=300000$ passages $(13 / 09 / 2012)$

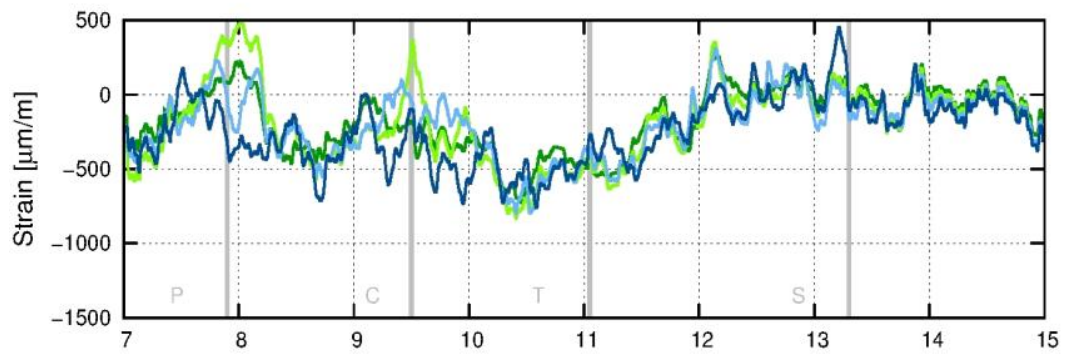

$\mathrm{N}=300000$ passages (04/12/2012) $\mathrm{N}=350000$ passages $(11 / 12 / 2012)$ $\mathrm{N}=400000$ passages $(18 / 12 / 2012)$ $\mathrm{N}=525000$ passages $(11 / 01 / 2013)$

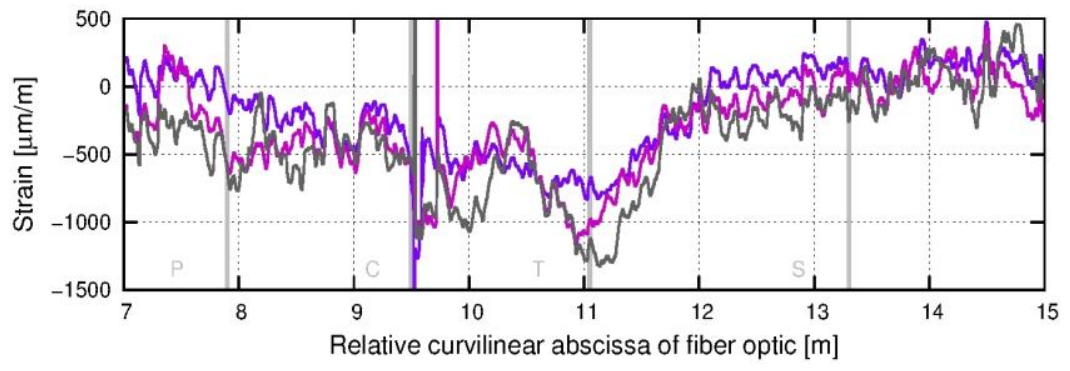

$\mathrm{N}=525000$ passages $(11 / 10 / 2013)$ $\mathrm{N}=625000$ passages $(22 / 11 / 2013)$ $\mathrm{N}=728000$ passages $(10 / 12 / 2013)$

Figure 5: Strains measured by the fiber optic at different load cycles

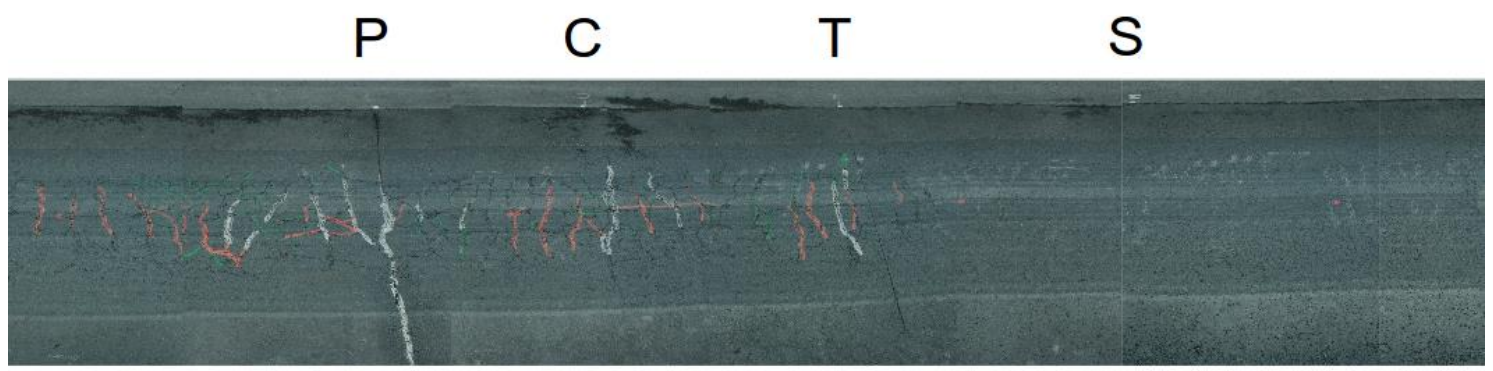

Figure 6: Photos of cracked pavement section at the end of the experimentation

At 525000 load cycles, the entire strain profile located between $x=7 \mathrm{~m}$ and $\mathrm{x}=12 \mathrm{~m}$ is strongly modified. This corresponds to the appearance and the growth of various cracks in this area (see figure 6). For the portion of the fiber optic located between $x=12 m$ and $x=15 m$, however, the change of the strain profile curves is less significant. This is in accordance with the fact that no cracks were detected by visual inspection for $\mathrm{x}>12 \mathrm{~m}$, until the end of the experimentation (see figure 6). So, these results show that the second measurement method, where no load is applied on the pavement, can also successfully detect the initiation of cracks or damage within the pavement, 
without requiring the application of a static load on the pavement. This second approach seems to be more appropriate to monitor long lengths of pavements.

\section{CONCLUSION}

A new non-destructive technique for the detection of cracking in asphalt pavements was tested at real scale on the pavement fatigue carrousel of IFSTTAR. It is based on the use of a fiber optic cable embedded in the pavement layer as distributed sensor. The fiber optic is interrogated by the Rayleigh sensing technique and from the evolution of the strain profiles measured at different time (after different levels of traffic) cracks can be detected.

In our study, we showed that two methods of post-treatment of the fiber optic measurements can be used. With both methods, significant changes of shape of the strain profiles could be directly linked to crack initiation and propagation. However, the method which does not require to apply a load on the pavement is the most promising, since it becomes possible to make measurements over relatively long distances of pavement $(70 \mathrm{~m}$ with the equipment used in this study, but more with other techniques).

It is planned to continue to evaluate the efficiency of the technique described in this paper. Indeed, the first results need to be confirmed for other pavement structures. Some additional tests will be performed on our pavement fatigue carrousel in the next months. A laboratory study is also planned, to carry out bending tests on pre-cracked asphalt plates, equipped with a continuous optical fiber. These tests, carried out in controlled conditions, should allow a better assessment of the sensitivity of the fiber to the presence of a crack in the asphalt, and of the influence of various parameters (loading time, temperature) on the results.

\section{REFERENCES}

[1] P. Autret, A.B. de Boissoudy, J.C. Gramsammer. The circular test track of the laboratoire central des ponts et chaussées (LCPC) Nantes - First Results, Proceedings of the $6^{\text {th }}$ International Conference on Asphalt Pavements, vol. 1, pp. 550-561. 1987.

[2] A. Güemes, A. Fernández-López, B. Soller. Optical Fiber Distributed Sensing - Physical Principles and Applications, Structural Health Monitoring, 9, pp. 233-245. 2010.

[3] X. Chapeleau, T. Sedran, L.M. Cottineau, J. Cailliau, F. Taillade, I. Gueguen, J.M. Henault. Study of ballastless track structure monitoring by distributed optical fiber sensors on a real-scale mockup in laboratory, Engineering Structures. 2013.

[4] J-M. Henault, J. Salin, G. Moreau, S. Delepine-Lesoille, J. Bertand, F. Taillade. Qualification of a truly distributed fiber optic technique for strain and temperature measurements in concrete structures, International workshop on ageing management of nuclear power plants and waste disposal structures, vol. 12. 2011.

[5] A. Kaplan, S. Klutte, D. Gifford, A. Heaney. Distributed optical fiber sensing for wind blade strain monitoring and defect detection, $8^{\text {th }}$ International Workshop on Structural Health Monitoring, Stanford (CA, USA). 2011.

[6] J.F. Corte, M.T. Goux. Design of pavement structures: the French technical guide, Transportation Research Record, vol. 1539, p.116-124. 1996. 